Acta Crystallographica Section F

Structural Biology

and Crystallization

Communications

ISSN 1744-3091

\section{Yongbin $\mathrm{Xu},{ }^{\mathrm{a}}$ Bo-Young Yun, Se-Hoon Sim, ${ }^{b}$ Kangseok Lee ${ }^{b}$ and Nam-Chul $\mathrm{Ha}^{\mathrm{a} *}$}

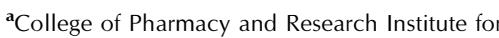
Drug Development, Pusan National University, Jangjeon-dong, Geumjeong-gu, Busan 609-735, Republic of Korea, and ${ }^{\mathbf{b}}$ Department of Life Science, Chung-Ang University, Seoul 156-756, Republic of Korea

Correspondence e-mail: hnc@pusan.ac.kr

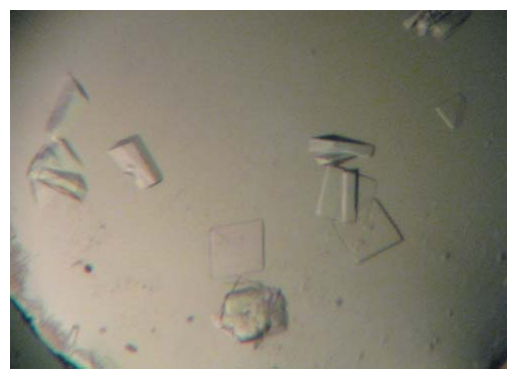

(C) 2009 International Union of Crystallography All rights reserved

\section{Crystallization and preliminary X-ray crystallographic analysis of Escherichia coli CusB}

Periplasmic membrane-fusion proteins (MFPs) are an essential component of multidrug and metal-efflux pumps in Gram-negative bacteria. However, the functional structure of MFPs remains unclear. CusCFBA, the $\mathrm{Cu}^{\mathrm{I}}$ and $\mathrm{Ag}^{\mathrm{I}}$ efflux system in Escherichia coli, consists of the MFP CusB, the OMF CusC and the RND-type transporter CusA. The MFP CusB bridges the inner membrane RND-type efflux transporter CusA and the outer membrane factor CusC and exhibits substrate-linked conformational changes which distinguish it from other MFP-family members. CusB from E. coli was overexpressed and the recombinant protein was purified using Ni-NTA affinity, Q anion-exchange and gel-filtration chromatography. The purified CusB protein was crystallized using the vapour-diffusion method. A diffraction data set was collected to a resolution of $3.1 \AA$ at $100 \mathrm{~K}$. The crystal belonged to space group $C 222$.

\section{Introduction}

Transition metals are crucial for every living cell (Nelson, 1999), but are toxic to the cell when present in excess amounts. In Gramnegative bacteria, copper is required for periplasmic and inner membrane enzymes and the primary target of copper-mediated damage appears to be the periplasm (Macomber et al., 2007). Thus, export of copper ions from the periplasm is necessary for bacteria to survive under excess metal conditions.

Periplasmic efflux pumps of the resistance-nodulation-division (RND) family are essential components in the intrinsic resistance of Gram-negative bacteria to lethal concentrations of drugs and metal ions (Poole \& Srikumar, 2001; Zgurskaya, 2002). The majority of RND-type efflux systems form a tripartite complex comprised of an energy-utilizing inner membrane transporter (Tseng et al., 1999), an outer membrane factor (Fralick, 1996) and a periplasmic membranefusion protein (MFP; Dinh et al., 1994). Among the RND-family efflux systems, the Escherichia coli AcrAB-TolC system, which is involved in the multidrug resistance of the bacteria, has been studied extensively (Bavro et al., 2008; Seeger et al., 2006; Murakami et al., 2006; Koronakis et al., 2000). However, the functional assembly of the AcrAB-TolC system remains under debate (Yum et al., 2009; Murakami et al., 2006; Xu et al., 2009).

The CusCFBA system is known as a $\mathrm{Cu}^{\mathrm{I}}$ and $\mathrm{Ag}^{\mathrm{I}}$ efflux pump in E. coli and consists of the MFP CusB, the OMF CusC and the RNDtype transporter CusA as indispensable components; this system is expected to have many structural and functional similarities to the AcrAB-TolC pump. Distinctively, CusF, a small periplasmic metalbinding protein, is only found in putative monovalent metal-ion resistance systems. The periplasmic MFP CusB is thought to bridge the inner membrane transporter and the outer membrane factor like the MFP AcrA in the multidrug-efflux pump AcrAB-TolC. However, recent studies have suggested that CusB is more likely to have a functional role in substrate capture and extrusion (Bagai et al., 2007). It has been proposed that CusB plays an active role in export through substrate-linked conformational changes. Indeed, a conformational change of CusB was induced by the binding of $\mathrm{Ag}^{\mathrm{I}}$, the substrate of the CusCFBA efflux pump (Bagai et al., 2007). In addition, CusB 
Table 1

Diffraction statistics.

(a) Data collection. Values in parentheses are for the highest resolution shell.

\begin{tabular}{ll}
\hline X-ray source & Beamline 4A, Pohang Accelerator Laboratory \\
Wavelength $(\AA)$ & 1.000 \\
Resolution $(\AA)$ & $50-3.1(3.21-3.10)$ \\
Unit-cell parameters $\left(\AA,^{\circ}\right)$ & $a=240.1, b=247.9, c=117.8, \alpha=\beta=\gamma=90$ \\
Completeness $(\%)$ & $95.2(75.3)$ \\
$R_{\text {merge }}^{\dagger}$ & $7.6 \%(40.2 \%)$ \\
Redundancy & $7.7(2.8)$ \\
Average $I / \sigma(I)$ & $15.5(1.6)$ \\
\hline
\end{tabular}

(b) Cell-content analysis.

\begin{tabular}{lllll}
\hline No. of molecules in ASU & 6 & 7 & 8 & 9 \\
\hline$V_{\mathrm{M}}\left(\AA^{3} \mathrm{Da}^{-1}\right)$ & 3.42 & 2.93 & 2.56 & 2.28 \\
Solvent content $(\%)$ & 64 & 58 & 52 & 46 \\
\hline
\end{tabular}

$\dagger R_{\text {merge }}=\sum_{h k l} \sum_{i}\left|I_{i}(h k l)-\langle I(h k l)\rangle\right| / \sum_{h k l} \sum_{i} I_{i}(h k l)$, where $I_{i}(h k l)$ and $\langle I(h k l)\rangle$ are the observed intensity and the mean intensity of related reflections, respectively.

naturally lacks lipid modification and thus is located in the periplasm, in contrast to other MFPs that are anchored to the inner membrane via lipid modification or an uncleavable signal sequence (Zgurskaya et al., 2009).

To date, three MFP structures have been determined after cleaving the membrane-anchoring moiety (Yum et al., 2009; Mikolosko et al., 2006; Akama et al., 2004; Higgins et al., 2004), but the oligomerization form of MFPs in their functional state remains to be elucidated. AcrA is believed to function as a trimer in vivo (Symmons et al., 2009), although the AcrA protein behaved as a monomer in solution (Zgurskaya \& Nikaido, 1999). However, MacA proteins, which are MFPs in a macrolide-specific efflux pump, have been crystallized as hexamers and the hexameric assembly was biochemically and genetically confirmed (Yum et al., 2009). Here, we report the crystallization and preliminary X-ray analysis of the mature form of CusB from E. coli. The CusB structure could provide insight into the active role of CusB in export through the substrate-linked conformational change and into how the MFPs assemble the efflux pump.

\section{Materials and methods}

2.1. DNA construction, protein expression and protein purification

A DNA fragment encoding E. coli CusB (residues 29-408; accession No. NC_00913) was amplified from the genomic DNA library of E. coli using the polymerase chain reaction. The DNA fragment was inserted into the NcoI and XhoI sites of the pPROEX-HTA (Invitrogen, USA) vector using the same procedure as reported for E. coli MacA (Yum et al., 2009; Piao et al., 2008). The resulting protein contained three additional amino acids (Gly-His-Met) between the TEV protease cleavage site and the mature protein as a cloning artifact. The recombinant CusB protein was expressed in E. coli BL21 (DE3) using LB medium supplemented with $50 \mu \mathrm{g} \mathrm{ml}^{-1}$ ampicillin at $310 \mathrm{~K}$ until the $\mathrm{OD}_{600}$ reached 0.5 . Protein expression was induced by adding $0.2 \mathrm{~m} M$ isopropyl $\beta$-D-1-thiogalactopyranoside (IPTG). The cells were harvested by centrifugation at $5000 \mathrm{~g}$ for $15 \mathrm{~min}$ at $277 \mathrm{~K}$. Harvested cells were suspended in lysis buffer containing $20 \mathrm{~m} M$ Tris $\mathrm{pH} 8.0$ and $150 \mathrm{mM} \mathrm{NaCl}$ and disrupted by sonication. After the cell debris had been removed by centrifugation at $45000 \mathrm{~g}$ for $30 \mathrm{~min}$, the resulting supernatant was loaded onto Ni-NTA agarose resin preequilibrated with lysis buffer. The resin was washed with lysis buffer supplemented with $20 \mathrm{~m} M$ imidazole and was then eluted with lysis buffer supplemented with $200 \mathrm{~m} M$ imidazole. The fractions con- taining CusB protein were pooled and $\beta$-mercaptoethanol was added to a final concentration of $10 \mathrm{~m} M$. This solution was incubated with recombinant TEV protease overnight at $277 \mathrm{~K}$ to remove the hexahistidine tag. The reaction mixture was subsequently loaded onto a $\mathrm{Q}$ anion-exchange column (Hitrap-Q; GE Healthcare, USA) for further purification and proteins were eluted from the column using a $0-1 M$ $\mathrm{NaCl}$ gradient in $20 \mathrm{~m} M$ Tris buffer $\mathrm{pH}$ 8.0. The collected fractions containing the CusB protein were pooled, concentrated and separated on a HiLoad Superdex 200 gel-filtration column (GE Healthcare, USA) pre-equilibrated with lysis buffer. During the purification, the presence of the protein was confirmed by SDS-PAGE. The purified protein was concentrated to $20 \mathrm{mg} \mathrm{ml}^{-1}$ in $20 \mathrm{~m} M$ Tris buffer pH 8.0 containing $150 \mathrm{mM} \mathrm{NaCl}$ and stored frozen at $193 \mathrm{~K}$ until use.

\subsection{Crystallization and data collection}

Initial crystallization of CusB was performed with commercially available screening solutions (Hampton Research, USA) using the microbatch method at various temperatures $(277,279,289$ and $295 \mathrm{~K})$. Crystals of the recombinant CusB protein were obtained by the vapour-diffusion technique at $279 \mathrm{~K}$. Crystals of CusB formed under several conditions but only at $279 \mathrm{~K}$. The crystallization conditions were optimized to produce shiny single crystals using the hanging-drop vapour-diffusion method at $279 \mathrm{~K}$ (Fig. 1). X-ray diffraction data from the crystals were collected using an ADSC Q310 CCD detector on beamline 4A of Pohang Light Source (PLS), South Korea.

\section{Results and discussion}

Although CusB has a signal sequence for export to the periplasm, we removed the signal sequence from the full-length protein when we generated the DNA construct for protein expression in the bacterial cytoplasm. Crystals suitable for data collection were obtained in droplets containing $1 \mu \mathrm{l}$ protein solution $\left[20 \mathrm{mg} \mathrm{m}^{-1}\right.$ protein in $20 \mathrm{~m} M$ Tris $\mathrm{pH} 8.0$ buffer containing $150 \mathrm{~m} M \mathrm{NaCl}$ ] and $1 \mu \mathrm{l}$ of a precipitant solution consisting of $0.2 \mathrm{M}$ sodium citrate tribasic, $0.1 \mathrm{M}$ Tris- $\mathrm{HCl} \mathrm{pH} 8.5$ and $20 \%(v / v)$ 2-propanol. The droplets were equilibrated by the hanging-drop vapour-diffusion method against $1 \mathrm{ml}$ precipitant solution at $279 \mathrm{~K}$ for a month. For X-ray data collection, a single crystal was briefly soaked in a cryoprotectant solution containing $0.2 \mathrm{M}$ sodium citrate tribasic, $0.1 \mathrm{M}$ Tris- $\mathrm{HCl} \mathrm{pH} 8.5$, $20 \%(v / v)$ 2-propanol and $25 \%(v / v)$ glycerol. A set of 360 images $(0-$ $360^{\circ}$ ) was obtained using a $1^{\circ}$ oscillation width and $30 \mathrm{~s}$ exposure time

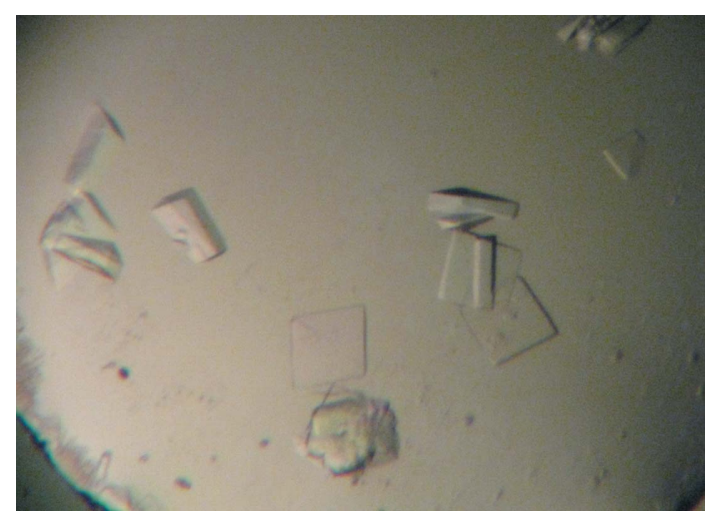

Figure 1

Crystals of E. coli CusB. Approximate dimensions are $0.2 \times 0.2 \times 0.05 \mathrm{~mm}$. 
at $1.00 \AA$ wavelength. The data collected were processed and scaled with the $H K L-2000$ package (Otwinowski \& Minor, 1997).

Based on the diffraction data, the crystal belonged to space group $C 222$, with unit-cell parameters $a=240.1, b=247.9, c=117.8 \AA$. In particular, analysis of diffraction along the $h, k$ and $l$ axes clearly demonstrated that all the axes of the crystal were rotational axes, not screw axes. The diffraction data set had a resolution range of 50$3.1 \AA$ with $95.2 \%$ completeness and an $R_{\text {merge }}$ of $7.6 \%$. Since the selfrotation function from the data set did not give any clue to the number of molecules per asymmetric unit, we were only able to estimate the putative number of molecules in the asymmetric unit from the calculated solvent content. Candidates for the number of molecules per asymmetric unit and the corresponding Matthews coefficients (Matthews, 1968) and solvent contents were calculated as listed in Table 1.

In order to solve the structure, molecular replacement was attempted using the structures of E. coli AcrA (29\% sequence similarity), E. coli MacA (21\% sequence similarity) and P. aeruginosa MexA (36\% sequence similarity) as search models in the program MOLREP (Collaborative Computational Project, Number 4, 1994), but this method was not successful. We are now attempting to use the MAD method to solve the phase problem using selenomethioninesubstituted crystals.

We thank Dr Yeon-Gil Kim for assistance at beamline 4A of PLS (Pohang, Korea). This work was supported by the 21C Frontier Microbial Genomics and Applications Center Program, MEST, Republic of Korea, and Seoul R\&BD program (10550).

\section{References}

Akama, H., Matsuura, T., Kashiwagi, S., Yoneyama, H., Narita, S., Tsukihara, T., Nakagawa, A. \& Nakae, T. (2004). J. Biol. Chem. 279, 25939-25942.
Bagai, I., Liu, W., Rensing, C., Blackburn, N. J. \& McEvoy, M. M. (2007). J. Biol. Chem. 282, 35695-35702.

Bavro, V. N., Pietras, Z., Furnham, N., Perez-Cano, L., Fernandez-Recio, J., Pei, X. Y., Misra, R. \& Luisi, B. (2008). Mol. Cell, 30, 114-121.

Collaborative Computational Project, Number 4 (1994). Acta Cryst. D50, 760-763.

Dinh, T., Paulsen, I. T. \& Saier, M. H. Jr (1994). J. Bacteriol. 176, $3825-$ 3831.

Fralick, J. A. (1996). J. Bacteriol. 178, 5803-5805.

Higgins, M. K., Bokma, E., Koronakis, E., Hughes, C. \& Koronakis, V. (2004). Proc. Natl Acad. Sci. USA, 101, 9994-9999.

Koronakis, V., Sharff, A., Koronakis, E., Luisi, B. \& Hughes, C. (2000). Nature (London), 405, 914-919.

Macomber, L., Rensing, C. \& Imlay, J. A. (2007). J. Bacteriol. 189, 16161626.

Matthews, B. W. (1968). J. Mol. Biol. 33, 491-497.

Mikolosko, J., Bobyk, K., Zgurskaya, H. I. \& Ghosh, P. (2006). Structure, 14, 577-587.

Murakami, S., Nakashima, R., Yamashita, E., Matsumoto, T. \& Yamaguchi, A. (2006). Nature (London), 443, 173-179.

Nelson, N. (1999). EMBO J. 18, 4361-4371.

Otwinowski, Z. \& Minor, W. (1997). Methods Enzymol. 276, 307-326.

Piao, S., Xu, Y. \& Ha, N.-C. (2008). Acta Cryst. F64, 391-393.

Poole, K. \& Srikumar, R. (2001). Curr. Top. Med. Chem. 1, 59-71.

Seeger, M. A., Schiefner, A., Eicher, T., Verrey, F., Diederichs, K. \& Pos, K. M. (2006). Science, 313, 1295-1298.

Symmons, M. F., Bokma, E., Koronakis, E., Hughes, C. \& Koronakis, V. (2009). Proc. Natl Acad. Sci. USA, 106, 7173-7178.

Tseng, T. T., Gratwick, K. S., Kollman, J., Park, D., Nies, D. H., Goffeau, A. \& Saier, M. H. Jr (1999). J. Mol. Microbiol. Biotechnol. 1, 107-125.

Xu, Y., Sim, S. H., Nam, K. H., Jin, X. L., Kim, H. M., Hwang, K. Y., Lee, K. \& Ha, N. C. (2009). Biochemistry, doi:10.1021/bi900415t.

Yum, S., Xu, Y., Piao, S., Sim, S. H., Kim, H. M., Jo, W. S., Kim, K. J., Kweon, H. S., Jeong, M. H., Jeon, H., Lee, K. \& Ha, N. C. (2009). J. Mol. Biol. 387, 1286-1297.

Zgurskaya, H. I. (2002). Int. J. Med. Microbiol. 292, 95-105.

Zgurskaya, H. I. \& Nikaido, H. (1999). J. Mol. Biol. 285, 409-420.

Zgurskaya, H. I., Yamada, Y., Tikhonova, E. B., Ge, Q. \& Krishnamoorthy, G. (2009). Biochim. Biophys. Acta, 1794, 794-807. 